\title{
Targeting intracellular degradation pathways for treatment of liver disease caused by $\alpha 1$-antitrypsin deficiency
}

\author{
Yan Wang ${ }^{1,2}$ and David H. Perlmutter ${ }^{1-3}$
}

The classic form of $\alpha 1$-antitrypsin deficiency (ATD) is a wellknown genetic cause of severe liver disease in childhood. A point mutation alters the folding of a hepatic secretory glycoprotein such that the protein is prone to misfolding and polymerization. Liver injury, characterized predominantly by fibrosis/ cirrhosis and carcinogenesis, is caused by the proteotoxic effect of polymerized mutant $\alpha 1$-antitrypsin Z (ATZ), which accumulates in the endoplasmic reticulum (ER) of hepatocytes. Several intracellular pathways have been shown to be responsible for disposal of ATZ after it accumulates in the ER, but autophagy appears to be specialized for disposal of insoluble ATZ polymers. Recently, we have found that drugs that enhance the activity of the autophagic pathway reduce the cellular load of mutant ATZ and reverse hepatic fibrosis in a mouse model of ATD. Because several of these autophagy enhancers have been used safely in humans for other reasons, we have been able to initiate a clinical trial of one of these drugs, carbamazepine, to determine its efficacy in severe liver disease due to ATD. In this review, we discuss the autophagy enhancer drugs as a new therapeutic strategy that targets cell biological mechanisms integral to the pathogenesis of liver disease due to ATD.

n 1963, exactly 50 years ago, Eriksson and Laurell first described a1-antitrypsin deficiency (ATD) when they used newly developed serum protein electrophoresis techniques and discovered that the usual al-globulin peak was missing from several patients with chronic obstructive pulmonary disease (COPD) (1). Much was learned about this condition in the next several years. First, it was found to be a relatively common genetic disease, with an incidence of 1 in 1,500-3,000 live births in many regions of the world (reviewed in ref. 2). The protein affected was found to be a secretory glycoprotein predominantly derived from the liver. Although it could inhibit trypsin in vitro, al-antitrypsin (AT) was found to be particularly effective in inhibiting neutrophil elastase and so its true physiological function is now thought to be inhibition of neutrophil elastase and several related neutrophil serine proteases. It is a highly regulated protein with serum levels rising three- to fivefold during the host response to inflammation/tissue injury (reviewed in ref. 3). Soon, it was also learned that there are several forms of ATD, including a classic form in which a point mutation leads to altered folding during biogenesis. In this classic form, the mutant protein, a1-antitrypsin Z (ATZ), accumulates in the early compartments of the secretory pathway, especially the endoplasmic reticulum (ER), while the amount of AT secreted is markedly reduced. Serum levels of AT in affected homozygotes are in the range of $10-15 \%$ of those in normal individuals (3). It did not take long to realize that susceptibility to emphysema/COPD is due to the proteolytic effect of uninhibited neutrophil proteases on the connective tissue matrix of the lung. This realization led to the protease-antiprotease paradigm for pathogenesis of COPD and to the rationale for use of replacement therapy with purified al-antitrypsin for patients with COPD due to ATD (reviewed in ref. 4).

In 1969, Sharp (5) made the curious observation that infants with cirrhosis were affected by the classic form of ATD. Soon, it was recognized that ATD was the most common genetic cause of liver disease in children and that it had a wide range of severity levels, including severe disease necessitating liver transplantation. It has taken many years to understand how this genetic defect causes liver disease, but we now know that the broad framework involves the proteotoxic effect of intracellular ATZ accumulation on the liver (reviewed in ref. 6). We also know that the tendency for misfolded ATZ to polymerize and aggregate in the ER of liver cells (Figure 1) plays a particularly important role in its proteotoxicity and the pathobiology of hepatic injury. Indeed, the proteotoxic effect of intracellular ATZ accumulation appears to have many characteristics in common with a class of diseases now attributed to "aggregation-prone" proteins such as amyloid- $\beta$ in Alzheimer's disease and the poly-Q proteins that cause Huntington's disease. In this article, we will review recent work on the cellular fate of mutant ATZ, how this influences the development of liver disease, and how it has led to novel therapeutic strategies, one of which has already reached a phase II/III clinical trial.

\section{Clinical Characteristics of ATD}

The clinical presentation of lung disease due to ATD is very similar to other causes of COPD. Interestingly, there is very little evidence for lung disease or dysfunction during childhood and 


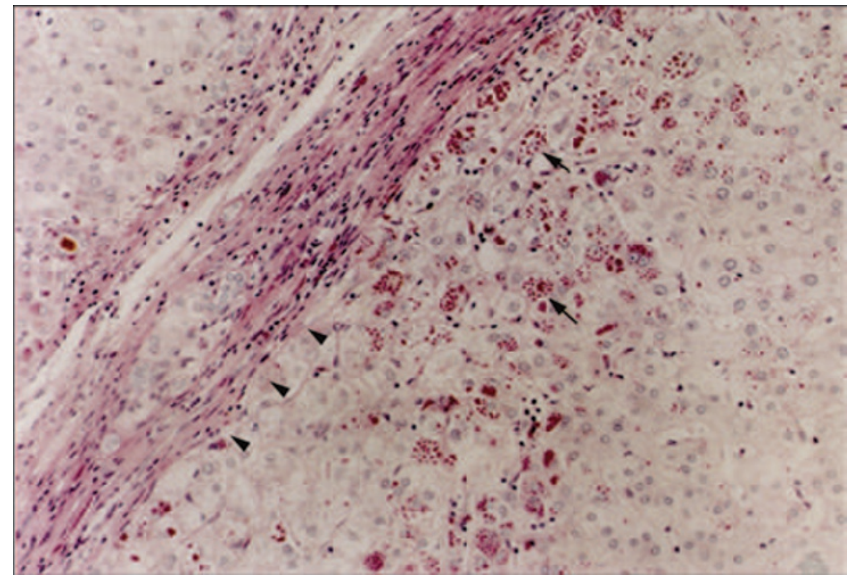

Figure 1. Histopathology of the liver in $\alpha 1$-antitrypsin deficiency. This liver section is stained with periodic acid-Schiff and then treated with diastase, showing the characteristic globules in hepatocytes (arrows) and a band of fibrosis (arrowheads).

adolescence. The most severe cases are discovered clinically in the mid to late 30s. Cigarette smoking increases the incidence and severity of lung disease in ATD by more than 1,000-fold (2).

There are reports that associate ATD with many other diseases, but almost all are limited in scope of experimental design, statistical analysis, and hypothetical mechanistic explanations that are not easy to reconcile with clinical observations. However, the curious association of ATD with vasculitis syndromes has recently been strengthened by genome-wide sequence analysis in a population of patients with antineutrophil cytoplasmic antibody-associated vasculitis, a population that includes what was previously known as Wegener's granulomatosis and microscopic polyangiitis (7).

Liver disease can present clinically in several different ways. The most common is jaundice in the neonatal period. In most cases, the jaundice resolves and there is no further evidence of liver dysfunction. Progressive liver dysfunction can develop in the first $4 \mathrm{y}$ of life, what we now call the "infantile" form of the disease. The "childhood" and "adolescent" forms of the disease present at 4-12 and 12-15 y of age with portal hypertension. In most of these cases, there is no history of neonatal jaundice. There is also a form of the disease that presents first at ages 40-70, and this "adult" form is much more common than previously recognized. In fact, this form of hepatic disease accounts for $88 \%$ of all liver transplants done for ATD in the United States (United Network for Organ Sharing (UNOS), personal communication). In all cases, the hepatic disease of ATD is characterized by fibrosis with relatively limited inflammation (3). ATD patients are also susceptible to hepatocellular carcinoma (8), usually developing in the adult form, but it has also been seen in adolescents (9).

In a unique cohort study, Sveger used nationwide screening in Sweden to identify 127 newborns with the classic form of ATD (10-12). The cohort has been followed for more than $40 \mathrm{y}$, and the results show that only $\sim 8 \%$ have experienced clinically significant liver disease. These results have led to the concept that genetic and/or environmental modifiers determine whether an individual with ATD falls within a subgroup that is susceptible to proteotoxicity or within the larger group of ATD patients who appear to be protected. Because the Sveger cohort has only reached ages in the fourth decade, the overall framework of this concept would only apply to the more extreme cases, namely, the infantile, childhood, and adolescent forms of ATD liver disease. Although there are many reports of liver disease in individuals heterozygous for the $Z$ allele, all of these reports involve populations that are biased in ascertainment, and susceptibility to liver disease in heterozygotes is still not definitively determined.

\section{Cellular Mechanisms That Determine Liver Disease}

The most important evidence that liver disease in ATD is caused by gain-of-function proteotoxicity comes from studies of mice transgenic for the mutant human ATZ gene. In each case, the mouse model develops liver disease even though its endogenous antielastases remain intact, and so the liver pathology cannot be caused by loss of function $(13,14)$. In the most well studied of these mouse models-the PiZ mousethe transgene was a genomic fragment of DNA that contains the coding regions of the ATZ gene together with introns and an $\sim 2-\mathrm{kb}$ portion of upstream and downstream flanking regions (13). The liver pathology of this mouse closely resembles the human liver pathology, with intrahepatocytic globules reflecting polymerized/aggregated ATZ accumulated in the ER, fibrosis, mild steatosis, hepatocyte-regenerative activity with mild inflammation, and increased incidence of hepatocellular carcinoma (15-17). Furthermore, it is characterized by morphological changes, including induced autophagosomes, structural alterations in mitochondria, and changes in signaling pathways that include, most notably, nuclear factor kappa-light-chain-enhancer of activated B cells (NF- $\kappa \mathrm{B})$ and transforming growth factor beta (TGF- $\beta$ ) activation $(18,19)$.

Because intracellular accumulation of ATZ and its proteotoxicity appear to be seminal for the pathogenesis of this liver disease and because so many homozygotes for ATD do not experience the clinical effects of hepatic proteotoxicity, we have focused our attention on the endogenous proteostasis mechanisms that could potentially counteract these types of damaging effects (Figure 2). First, we directed our studies to elucidating the mechanisms by which liver cells degrade mutant ATZ and discovered that the proteasomal and autophagy pathways play a major role (reviewed in ref. 20). The proteasome, as a part of the process that is called ER-associated degradation, is probably responsible for degradation of soluble monomeric ATZ, whereas autophagy is specialized for degradation of ATZ polymers and aggregates that can be either soluble or insoluble. Autophagy is a ubiquitous pathway by which cells generate amino acids to survive stress conditions by digesting internal constituents. It is characterized by double membrane vacuoles that form in the cytoplasm, sequestering cytosol and organelles and then fusing with lysosomes for degradation of the internal constituents of the "autophagic" vacuole. The autophagic pathway has been shown to play a critical role in degradation of aggregated proteins, and the known decline in autophagy 


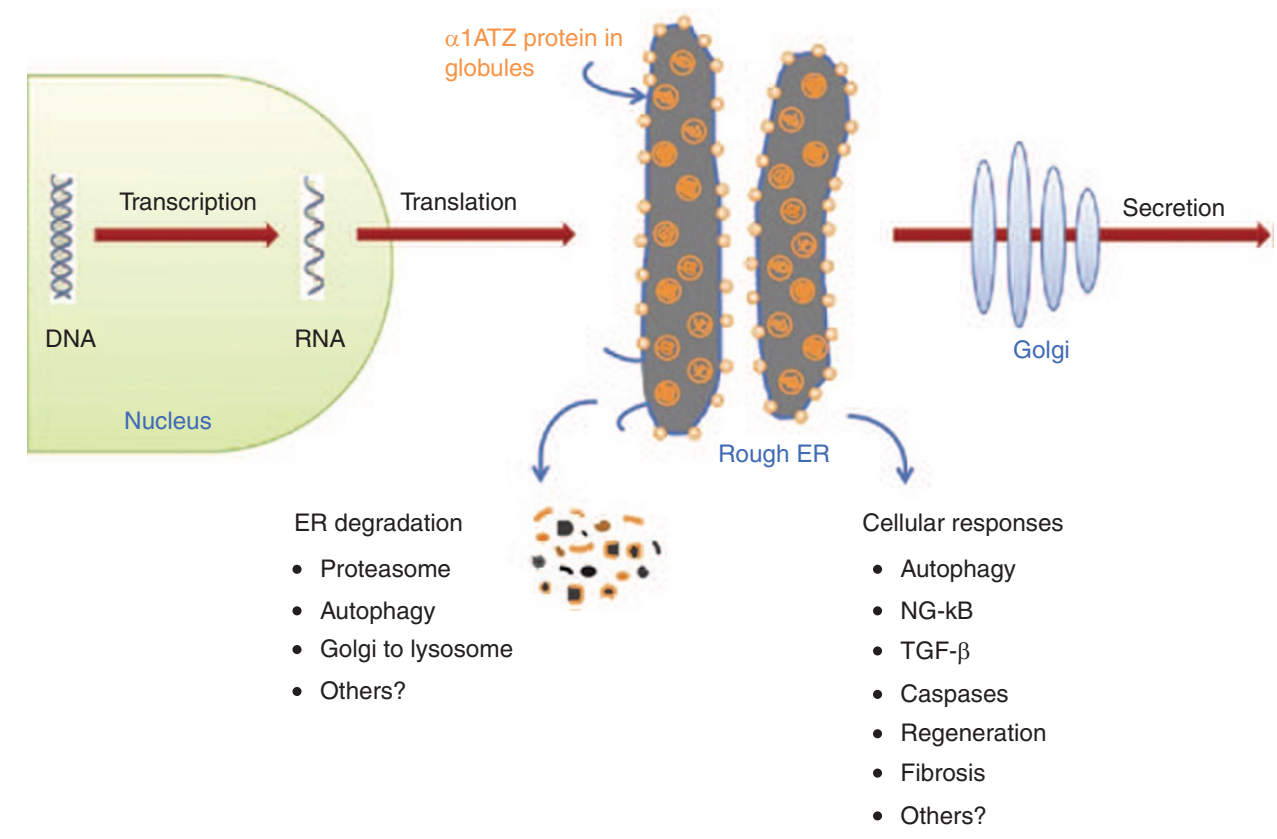

Figure 2. Endogenous proteostasis mechanism activated by accumulation of mutant $\alpha 1$-antitrypsin $Z$ in the endoplasmic reticulum (ER). ER degradation pathways are shown on the left and cellular responses on the right.

function with aging has thus been implicated in the pathogenesis of age-dependent diseases caused by aggregation-prone proteins and aging itself (reviewed in ref. 21). Recently, a pathway from Golgi complex to lysosome that involves sortilin has been shown to play a role in intracellular degradation of ATZ (22), and we suspect that there are additional mechanisms that have not yet been identified by which cells degrade ATZ.

To determine how cells might mitigate the proteotoxicity of intracellular ATZ accumulation and presumably protect ATD hosts from liver disease, we also focused on signaling pathways that activate proteostasis mechanisms. Using a variety of genetically engineered mammalian cell line and mouse models, particularly models with inducible expression of ATZ, we have found that there is a distinct set of signaling pathways that are activated when ATZ accumulates in cells. NF- $\kappa B$, TGF- $\beta$, ER caspases, mitochondrial caspases, and the autophagic response appear to be the key signaling pathways activated in model systems and liver tissue from ATD patients $(18,19)$. The NF- $\kappa B$ pathway probably plays a role in the effect of ATZ accumulation on hyperproliferation and carcinogenesis, and the TGF- $\beta$ pathway is likely to be a major determinant of the fibrotic response that is characteristic of ATZ proteotoxicity. Activation of ER caspase-12 and mitochondrial caspases is likely to be involved in the alterations of mitochondrial structure and function that have been found in response to ATZ proteotoxicity $(18,23)$. Activation of the autophagic response was shown when a mouse model with hepatocyte-specific inducible expression of ATZ, the $\mathrm{Z}$ mouse, was bred onto a genetic background that generates green fluorescent autophagosomes, the green fluorescent protein (GFP)-LC3 mouse (24). In contrast to the GFP-LC3 mouse in which starvation was necessary to induce GFP+ autophagosomes in the liver, induction of ATZ gene expression was sufficient to induce hepatic GFP+ autophagosomes in the
$Z \times$ GFP-LC3 mice in the absence of starvation. This particular result indicates that when ATZ accumulates in cells in an ATD patient, it specifically activates autophagy, and then autophagy participates in the disposal of ATZ. It is also notable that activation of the unfolded protein response is impaired in mammalian ATD model systems and tissues from ATD patients $(18,19)$. Although the mechanism for this impairment is not elucidated, we suspect it is an important part of the hepatic pathobiology, perhaps accounting for why globule-containing hepatocytes are found in the liver and drive the hyperproliferative state rather than undergoing apoptosis because of chronic unfolded protein response activation.

\section{Carbamazepine Promotes Autophagic Degradation of ATZ and Reduces Hepatic Proteotoxicity in the PiZ Mouse Model}

One of the major reasons that we investigated the intracellular degradation mechanisms and signaling pathways that play a role in the fate of ATZ is because we predicted that these would be targets of disease modifiers and potential targets of novel therapeutic strategies. To address this prediction, we recently investigated the possibility that autophagy enhancer drugs mitigate the proteotoxicity of ATZ and could therefore be used as a therapeutic strategy. We selected autophagy enhancer drugs because autophagy appears to be particularly important in ATD in that it is both specifically activated and plays a role in ATZ disposal. Furthermore, at the time we initiated the investigation, several drugs were shown to enhance autophagic degradation of aggregation-prone proteins, particularly the poly-Q proteins $(25,26)$. From this list of autophagy enhancer drugs, we focused on carbamazepine (CBZ), an anticonvulsant and mood stabilizer, because it has been used very extensively in clinical practice. The results showed that CBZ could indeed mediate increased intracellular degradation of ATZ but, even 
more importantly, the results showed that when administered orally to PiZ mice, the drug mediated a reduction in both hepatic ATZ load and hepatic fibrosis (15). Because CBZ is approved by the US Food and Drug Administration for use in clinical practice, it has been moved into a phase II/III clinical trial for patients with severe liver disease due to ATD. The results of this study also validate the concept that endogenous proteostasis mechanisms, mechanisms that probably protect ATD patients from hepatic proteotoxicity and disease, can be targeted for effective drug therapy (Figure 3).

\section{New Autophagy Enhancer Drugs Discovered by Automated Screening Using a Caenorhabditis elegans Model of ATD}

Recently, a transgenic C. elegans strain was developed as a novel animal model of ATD. A chimeric GFP-ATZ plasmid was used as the transgene, and it was targeted for expression in the intestine because intestinal cells of $C$. elegans carry out many of the functions attributed to the liver in higher organisms. The model recapitulates the cellular defect of ATD with intracellular accumulation of GFP-ATZ associated with proteotoxicity, as shown by slowing of larval development, decreased brood size, and diminished longevity. The model has also been adapted to automated high-content screening platform using an automated array scanner that detects the GFP+ ATZ accumulation. Screening of drug libraries using this platform has provided further validation for the autophagy enhancer class of drugs and has suggested some additional concepts for drug development. An initial screen of the Library of Pharmacologically
Active Compounds (LOPAC) drug library identified five hit compounds that mediated dramatic, dose-dependent reductions in ATZ load (27). Interestingly, four of these five hit compounds have the property of enhancing autophagy. These four compounds are all in active clinical use and so, similar to CBZ, they can immediately be tested in clinical trials, "repurposing" them for ATD. Another very interesting aspect of these findings is that two of the compounds are from the phenothiazine family, a drug family that is structurally related to tricyclic antidepressants, including CBZ. The phenothiazines have also been shown to enhance autophagic degradation of the aggregation-prone protein huntingtin that causes Huntington's disease $(25,28)$. Thus, this type of screening platform provides a wonderful new model for drug discovery for ATD and two new strategies for chemical- and computation-based drug discovery using the autophagy enhancer drug paradigm and the phenothiazine structure.

\section{New Autophagy Enhancer Drugs Arising From Other Drug Discovery Platforms}

A number of other autophagy enhancer drugs have been described in the recent literature (Figure 4). For example, fluspirilene, another phenothiazine that was identified by high-throughput screening for autophagic degradation of huntingtin (25), has been investigated in further detail (29). The studies show that fluspirilene induces autophagy by reducing intracellular $\mathrm{Ca}^{2+}$ and preventing calpain 1-mediated cleavage of autophagy gene ATG5. Higher levels of uncleaved ATG5

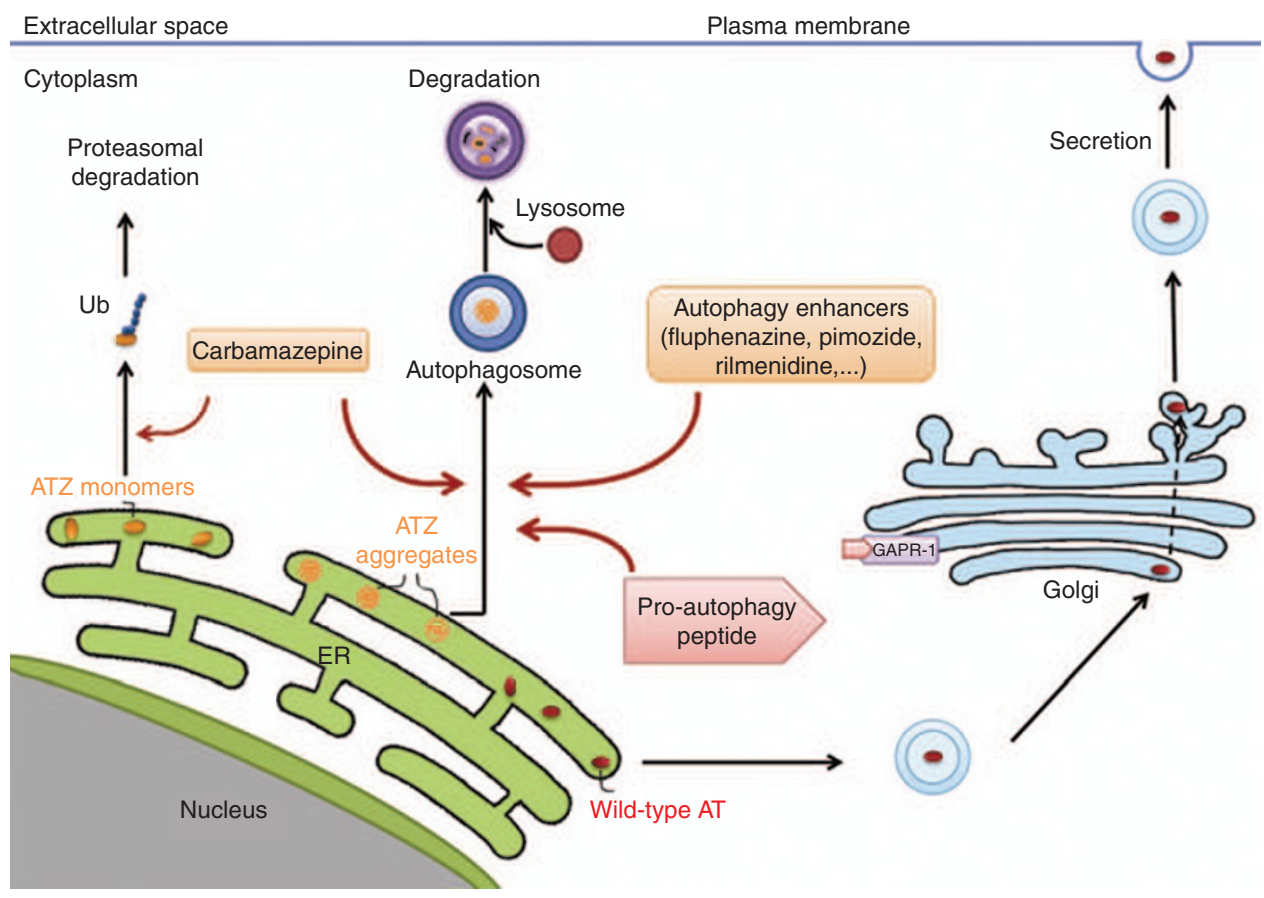

Figure 3. Site of action of autophagy enhancer drugs. The normal secretory pathway traversed by wild-type $\alpha 1$-antitrypsin (AT) is shown at the bottom and then at the far right edge of the figure. $\alpha 1$-Antitrypsin Z (ATZ) aggregates (yellow) being degraded by autophagy are shown in the middle. ATZ monomers being degraded by the ubiquitin (Ub)-dependent proteasomal pathway are shown on the left. Carbamazepine enhances both proteasomal degradation and autophagic degradation. Based on the current data, other autophagy enhancers, including fluphenazine, pimozide, and rilmenidine, enhance autophagic degradation. Proautophagy peptide Tat-beclin-1 binds to GAPR-1 (Golgi-associated plant pathogenesis-related protein 1, which is a Golgi-associated autophagy inhibitor) and releases beclin-1 from Golgi, thereby increasing free beclin-1 in the cytoplasm to induce autophagy. 


\section{Autophagy enhancer drugs for ATD $\quad$ Review}

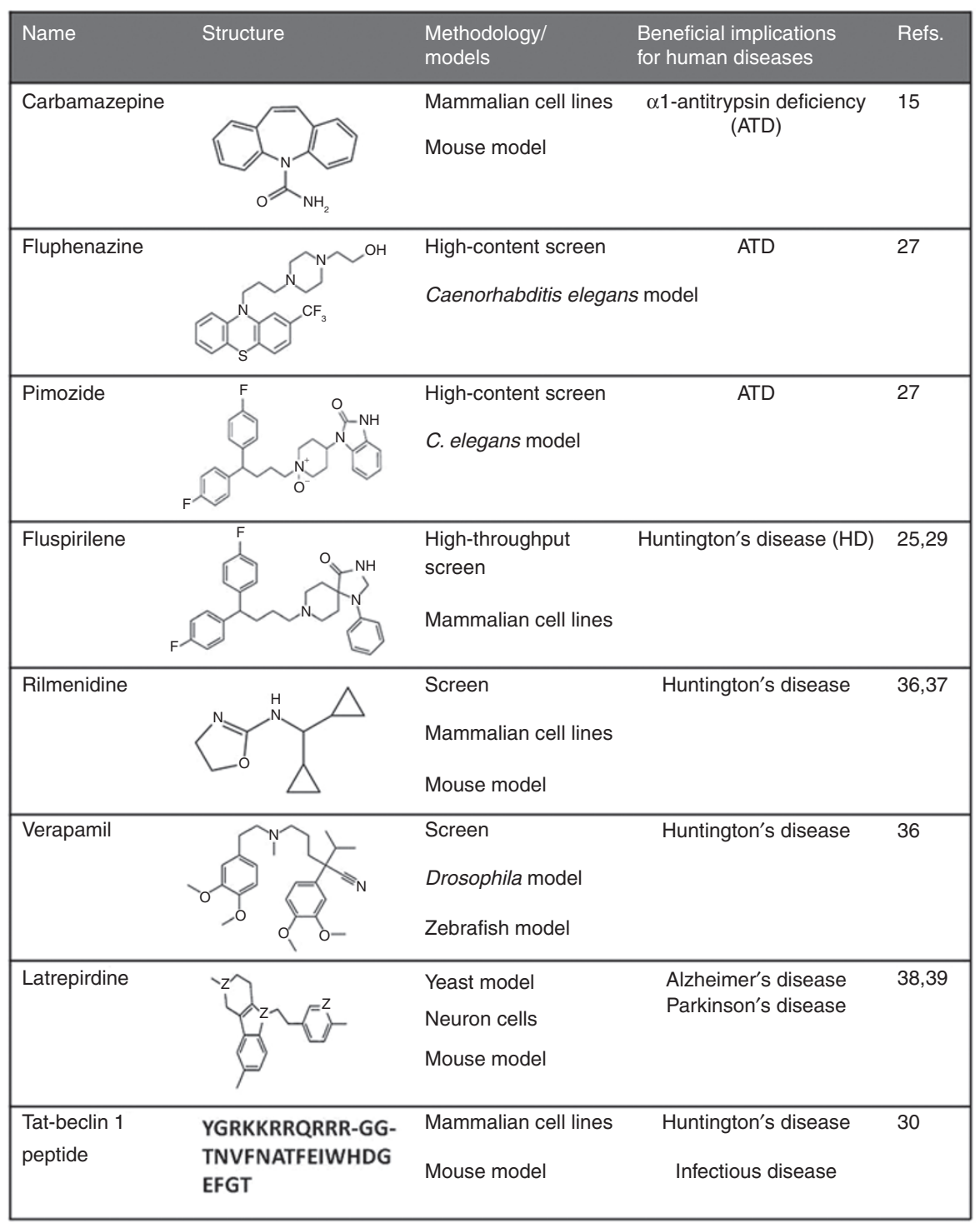

Figure 4. Selected autophagy enhancers and their beneficial implications for human diseases. The structure of each drug, how it was evaluated, diseases being targeted, and references are shown.

were associated with increased formation of autophagosomes. This is exciting because fluspirilene is a drug approved by the US Food and Drug Administration and because it may provide a mechanistic explanation for the effect of the phenothiazine drug class on autophagy.

A novel autophagy-inducing peptide has recently been identified, and it has potential applications in degradation of protein aggregates and in treating infectious disease (30). Previous studies have shown that the human immunodeficiency virus (HIV) protein Nef acts as an autophagy inhibitor by directly interacting with the autophagy regulatory factor beclin-1 (31). Shoji-Kawata took advantage of this information about HIV by designing a peptide based on the Nef-binding region of beclin- 1 . The Tat sequence was engineered into the peptide to provide for cell penetration. This Tat beclin-1 peptide had powerful autophagy-inducing activity, as evidenced by the degradation of mutant huntingtin and antagonistic activity against several invasive bacterial and viral pathogens. It was found to bind to Golgi-associated plant pathogenesis-related protein 1, a previously unknown negative regulator of autophagy identified in this work. The competing interaction of Tat-beclin-1 to Golgiassociated plant pathogenesis-related protein 1 releases beclin-1 from the Golgi complex, thereby increasing free beclin-1 in the cytosol to induce autophagy. This autophagy-inducing peptide would therefore be a potential therapeutic candidate for ATD.

A novel gene therapy approach to enhance autophagy has recently been described by Pastore et al. (32). This study reported that transcription factor EB (TFEB), a master gene that regulates autophagy and lysosomal gene expression, induced autophagy-dependent ATZ clearance in a mammalian cell line model. Using adenovirus-mediated gene transfer of TFEB in the PiZ mouse model of ATD, this strategy was shown to significantly promote hepatic ATZ clearance and reduce liver fibrosis in vivo. Although it will require more time for this strategy to gain entry into clinical trials than the drugs approved by the US Food and Drug Administration mentioned 
above, the results of this study provide further validation for upregulation of autophagy as a therapeutic approach for ATD liver disease.

\section{Enhancement of Autophagy as a Therapeutic Strategy for Other Diseases}

An important feature of autophagy is that it recycles amino acids, lipids, and other metabolic precursors, and these serve as nutrients for energy metabolism and maintenance of essential cellular functions. Under stress conditions, enhanced autophagy allows cells to adapt to changing nutritional and energy demands through protein catabolism (reviewed in ref. 33). He et al. (34) recently showed that acute exercise induces autophagy in skeletal and cardiac muscle, adipose tissue, and pancreatic $\beta$ cells in mouse models. Interestingly, mice that have exercise-induced autophagy in these tissues are more resistant to high-fat-diet-induced glucose intolerance, leptin resistance, and increased levels of serum cholesterol and triglycerides. These exercise-associated benefits are not seen in the BCL2 AAA mutant mice that are deficient in exerciseinduced autophagy. Thus, this result indicates that autophagy enhancers may have beneficial effects on age-dependent metabolic diseases, such as type 2 diabetes.

Most neurodegenerative disease-associated proteins that form aggregates or inclusions are autophagy substrates. Enhanced autophagy decreases the toxic accumulation of these mutant proteins, such as mutant huntingtin (Huntington's disease), mutant $\alpha$-synuclein (Parkinson's disease), mutant ataxin-3 (spinocerebellar ataxia type 3) and tau (Alzheimer's disease; reviewed in ref. 35). Therefore, autophagy enhancers have been extensively studied for their potential to treat neurodegenerative diseases. For example, a screen of drugs approved by the US Food and Drug Administration identified five drugs that enhance autophagy through mammalian target of rapamycin (mTOR)-independent pathways and, thereby, enhance clearance of mutant huntingtin in mechanistic cell lines and models of Drosophila and zebrafish (36). These drugs are $\mathrm{K}^{+}$channel opener minoxidil, Gi-signaling activator clonidine and rilmenidine, and L-type $\mathrm{Ca}^{2+}$ channel antagonists verapamil, amiodarone, and loperamide. A followup study has shown that rilmenidine induces autophagy in primary neuronal cultures and alleviates toxicity of polyglutamine expansion in a transgenic mouse model for Huntington's disease (37). This result suggests that rimenidine, a safe antihypertensive drug, is a promising candidate for clinical trials in Huntington disease. The drug latrepirdine has recently been shown to enhance autophagic degradation of $\alpha$-synuclein in a cell line model of Parkinson's disease (38) and reverses cognitive decline in a mouse model of Alzheimer's disease (39).

Autophagy also plays a critical role in the immune system. Many medically important pathogens (varieties of bacteria and viruses) are degraded by autophagy (reviewed in ref. 35). Thus, autophagy enhancer strategies may have therapeutic potential for infectious diseases. For example, stimulation of autophagy by vitamin $\mathrm{D}$ was implicated as a strategy for inhibiting Mycobacterium tuberculosis infection (40). The Tat-beclin-1 peptide, which activates autophagy, was shown to have antiinfective activity in mammalian cell lines when tested against infection from three positive-stranded RNA viruses (sindbis virus (SINV), chikungunya virus (CHIKV), West Nile virus. (WNV)), human immunodeficiency virus (HIV)-1, and the intracellular bacterium, Listeria monocytogenes (30).

In summary, the discovery of several pharmacological strategies for enhancing autophagy holds great promise for therapeutic intervention, including the liver disease caused by ATD. This strategy is particularly appealing because it targets a fundamental cell biological mechanism and one of the major mechanisms by which the cell protects itself from proteotoxicity.

\section{STATEMENT OF FINANCIAL SUPPORT}

We are grateful for grants from the National Institutes of Health (Bethesda, MD; DK076918, DK084512, and DK096990) and for institutional funds from the Children's Hospital of Pittsburgh of the University of Pittsburgh Medical Center, which have supported our studies reported here.

Disclosure: The authors have no financial conflicts to disclose.

\section{REFERENCES}

1. Laurell C-B, Erikkson S. The electrophoretic alpha-1-globulin pattern of serum in alpha-1-antitrypsin deficiency. Scand J Clin Lab Invest 1963;15:132-40.

2. Silverman EK, Sandhaus RA. Clinical practice. Alpha1-antitrypsin deficiency. N Engl J Med 2009;360:2749-57.

3. Perlmutter DH. a1-antitrypsin deficiency. In: Suchy FJ, Sokol RJ, Balistreri WF, eds. Liver Disease in Children, 3rd edn. Philadelphia, PA: Lippincott Williams \& Wilkins, 2007:545-71.

4. Crystal RG. Alpha 1-antitrypsin deficiency, emphysema, and liver disease. Genetic basis and strategies for therapy. J Clin Invest 1990;85:1343-52.

5. Sharp HL, Bridges RA, Krivit W, Freier EF. Cirrhosis associated with alpha1-antitrypsin deficiency: a previously unrecognized inherited disorder. J Lab Clin Med 1969;73:934-9.

6. Perlmutter DH, Silverman GA. Hepatic fibrosis and carcinogenesis in alantitrypsindeficiency: a prototype for chronic tissue damage in gain-offunction disorders. Cold Spring Harb Perspect Biol 2011;3:181-94.

7. Lyons PA, Rayner TF, Trivedi S, et al. Genetically distinct subsets within ANCA-associated vasculitis. N Engl J Med 2012;367:214-23.

8. Eriksson S, Carlson J, Velez R. Risk of cirrhosis and primary liver cancer in alpha 1-antitrypsin deficiency. N Engl J Med 1986;314:736-9.

9. Hadzic N, Quaglia A, Mieli-Vergani G. Hepatocellular carcinoma in a 12-year old child with PiZZ alpha-1-antitrypsin deficiency. Hepatol 2006;43:194.

10. Sveger T. Liver disease in alphal-antitrypsin deficiency detected by screening of 200,000 infants. N Engl J Med 1976;294:1316-21.

11. Piitulainen E, Carlson J, Ohlsson K, Sveger T. Alpha1-antitrypsin deficiency in 26-year-old subjects: lung, liver and protease/protease inhibitor studies. Chest 2005;128:2076-81.

12. Janciauskiene S, Wallmark A, Piitulainen E, Köhnlein T, Welte T, Sveger T. Performance of enhanced liver fibrosis plasma markers in asymptomatic individuals with ZZ a1-antitrypsin deficiency. Eur J Gastroenterol Hepatol 2011;23:716-20.

13. Carlson JA, Rogers BB, Sifers RN, et al. Accumulation of PiZ alpha 1-antitrypsin causes liver damage in transgenic mice. J Clin Invest 1989;83:1183-90.

14. Dycaico MJ, Grant SG, Felts K, et al. Neonatal hepatitis induced by alpha 1-antitrypsin: a transgenic mouse model. Science 1988;242:1409-12.

15. Hidvegi T, Ewing M, Hale P, et al. An autophagy-enhancing drug promotes degradation of mutant alphal-antitrypsin $\mathrm{Z}$ and reduces hepatic fibrosis. Science 2010;329:229-32.

16. Rudnick DA, Liao Y, An JK, Muglia LJ, Perlmutter DH, Teckman JH. Analyses of hepatocellular proliferation in a mouse model of alpha-1-antitrypsin deficiency. Hepatology 2004;39:1048-55. 


\section{Autophagy enhancer drugs for ATD Review}

17. Marcus NY, Brunt EM, Blomenkamp K, et al. Characteristics of hepatocellular carcinoma in a murine model of alpha-1-antitrypsin deficiency. Hepatol Res 2010;40:641-53.

18. Hidvegi T, Schmidt BZ, Hale P, Perlmutter DH. Accumulation of mutant alpha1-antitrypsin $\mathrm{Z}$ in the endoplasmic reticulum activates caspases-4 and -12, NFkappaB, and BAP31 but not the unfolded protein response. J Biol Chem 2005;280:39002-15.

19. Hidvegi T, Mirnics K, Hale P, Ewing M, Beckett C, Perlmutter DH. Regulator of Gsignaling 16 is a marker for the distinct ER stress state associated with aggregated mutant $\alpha 1$-antitrypsin $\mathrm{Z}$ in the classical form of $\alpha 1$ antitrypsin deficiency. J Biol Chem 2007;282:27769-80.

20. Perlmutter DH. Alpha-1-antitrypsin deficiency: importance of proteasomal and autophagic degradative pathways in disposal of liver diseaseassociated protein aggregates. Annu Rev Med 2011;62:33-345.

21. Rubinsztein DC, Mariño G, Kroemer G. Autophagy and aging. Cell 2011;146:682-95.

22. Gelling CL, Dawes IW, Perlmutter DH, Fisher EA, Brodsky JL. The endosomal protein sorting receptor sortilin has a role in trafficking $\alpha-1$ antitrypsin. Genetics 2012;192:889-903.

23. Teckman JH, An JK, Blomenkamp K, Schmidt B, Perlmutter DH. Mitochondrial autophagy and injury in the liver in alpha-1-antitrypsin deficiency. Am J Physiol 2004;286:G851-G862.

24. Kamimoto T, Shoji S, Hidvegi T, et al. Intracellular inclusions containing mutant alpha1-antitrypsin $\mathrm{Z}$ are propagated in the absence of autophagic activity. J Biol Chem 2006;281:4467-76.

25. Zhang L, Yu J, Pan H, et al. Small molecule regulators of autophagy identified by an image-based high-throughput screen. Proc Natl Acad Sci USA 2007;104:19023-8.

26. Sarkar S, Perlstein EO, Imarisio S, et al. Small molecules enhance autophagy and reduce toxicity in Huntington's disease models. Nat Chem Biol 2007;3:331-8.

27. Gosai SJ, Kwak JH, Luke CJ, et al. Automated high-content live animal drug screening using $\mathrm{C}$. elegans expressing the aggregation prone serpin a1-antitrypsin Z. PLoS ONE 2010;5:e15460.

28. Tsvetkov AS, Miller J, Arrasate M, Wong JS, Pleiss MA, Finkbeiner S. A small-molecule scaffold induces autophagy in primary neurons and protects against toxicity in a Huntington disease model. Proc Natl Acad Sci USA 2010;107:16982-7.

29. Xia HG, Zhang L, Chen G, et al. Control of basal autophagy by calpain 1 mediated cleavage of ATG5. Autophagy 2010;6:61-6.

30. Shoji-Kawata S, Sumpter R, Leveno M, et al. Identification of a candidate therapeutic autophagy-inducing peptide. Nature 2013;494:201-6.

31. Kyei GB, Dinkins C, Davis AS, et al. Autophagy pathway intersects with HIV-1 biosynthesis and regulates viral yields in macrophages. J Cell Biol 2009;186:255-68.

32. Pastore N, Blomenkamp K, Annunziata F, et al. Gene transfer of master autophagy regulator TFEB results in clearance of toxic protein and correction of hepatic disease in alpha-1-anti-trypsin deficiency. EMBO Mol Med 2013;5:397-412.

33. Kuma A, Mizushima N. Physiological role of autophagy as an intracellular recycling system: with an emphasis on nutrient metabolism. Semin Cell Dev Biol 2010;21:683-90.

34. He C, Bassik MC, Moresi V, et al. Exercise-induced BCL2-regulated autophagy is required for muscle glucose homeostasis. Nature 2012;481:511-5.

35. Rubinsztein DC, Codogno P, Levine B. Autophagy modulation as a potential therapeutic target for diverse diseases. Nat Rev Drug Discov 2012;11:709-30.

36. Williams A, Sarkar S, Cuddon P, et al. Novel targets for Huntington's disease in an mTOR-independent autophagy pathway. Nat Chem Biol 2008;4:295-305.

37. Rose C, Menzies FM, Renna M, et al. Rilmenidine attenuates toxicity of polyglutamine expansions in a mouse model of Huntington's disease. Hum Mol Genet 2010;19:2144-53.

38. Steele JW, Gandy S. Latrepirdine (Dimebon ${ }^{\circ}$ ), a potential Alzheimer therapeutic, regulates autophagy and neuropathology in an Alzheimer mouse model. Autophagy 2013;9:617-8.

39. Steele JW, Ju S, Lachenmayer ML, et al. Latrepirdine stimulates autophagy and reduces accumulation of a-synuclein in cells and in mouse brain. Mol Psychiatry 2013;18:882-8.

40. Campbell GR, Spector SA. Vitamin D inhibits human immunodeficiency virus type 1 and Mycobacterium tuberculosis infection in macrophages through the induction of autophagy. PLoS Pathog 2012;8:e1002689. 